\title{
Regeneration of the retina: toward stem cell therapy for degenerative retinal diseases
}

\author{
Sohee Jeon ${ }^{1,2} \mathcal{E}$ Il-Hoan $\mathrm{Oh}^{1, *}$ \\ ${ }^{1}$ Catholic High-Performance Cell Therapy Center \& Department of Medical Lifescience, ${ }^{2}$ Department of Ophthalmology, Seoul St. Mary's \\ Hospital, The Catholic University of Korea, Seoul 137-701, Korea
}

\begin{abstract}
Degenerative retinal diseases affect millions of people worldwide, which can lead to the loss of vision. However, therapeutic approaches that can reverse this process are limited. Recent efforts have allowed the possibility of the stem cell-based regeneration of retinal cells and repair of injured retinal tissues. Although the direct differentiation of pluripotent stem cells into terminally differentiated photoreceptor cells comprises one approach, a series of studies revealed the intrinsic regenerative potential of the retina using endogenous retinal stem cells. Muller glial cells, ciliary pigment epithelial cells, and retinal pigment epithelial cells are candidates for such retinal stem cells that can differentiate into multiple types of retinal cells and be integrated into injured or developing retina. In this review, we explore our current understanding of the cellular identity of these candidate retinal stem cells and their therapeutic potential for cell therapy against degenerative retinal diseases. [BMB Reports 2015; 48(4): 193-199]
\end{abstract}

\section{RETINAL DEGENERATIVE DISEASE AND SEARCH FOR RETINAL REGENERATION}

Degenerative retinal diseases are a heterogeneous group of eye diseases that can eventually cause permanent visual loss. Currently, millions of patients worldwide suffer from degenerative retinal diseases (1). Damage to any type of retinal neuron results in irreversible changes, and therapeutic modalities that can reverse these degenerative processes have not been available. However, recent progress in stem cell research has provided emerging hope for visual restoration in degenerative retinal diseases using cellular therapeutic approaches. One representative example of such a possibility was provided by a recent study on the transplantation of retinal pigment epithelial

*Corresponding author. Tel: +82-2-2258-8268; Fax: +82-2-591-3994; E-mail: iho@catholic.ac.kr

http://dx.doi.org/10.5483/BMBRep.2015.48.4.276

Received 5 November 2014

Keywords: Ciliary pigment epithelial cells, Muller glial cells, Retina, Retinal pigment epithelial cells, Stem cell
(RPE) cells that were derived from embryonic stem cells (ESCs) (2). A similar trial is underway by a Japanese stem cell research group using induced pluripotent stem cells (iPSCs).

Interestingly, both clinical studies target Stargardt's macular dystrophy and dry age-related macular degeneration, in which RPE cell loss is the major pathophysiology. Although the molecular mechanisms that underlie various degenerative retinal diseases vary, they share a common endpoint: the irreversible loss of photoreceptor cells. Therefore, the transplantation of photoreceptor cells is mandatory for visual restoration in most degenerative retinal diseases. Accordingly, developing strategies for the regeneration of photoreceptor cells has been a highlighted area of interest in ophthalmology and regenerative medicine. Studies have identified two major sources of stem cells for photoreceptors: pluripotent stem cells (e.g., ESCs and iPSCs) and tissue-specific stem cells in retinal tissue (3-9). Although the multipotential differentiation of pluripotent stem cells (ESCs or iPSCs) allows the possibility of specific differentiation into photoreceptor cells, little success have been reported in this area of research, mostly because of procedural difficulties and such risk factors as tumor formation and viral integration. On the other hand, the possibility that endogenous retinal stem cells can be used for retinal regeneration has been suggested by several lines of investigation using retinal injury models (3-7). However, unlike other types of well-defined tissue-specific stem cells in other organs in the body, identification of the cells responsible for the retinal regenerative process has been hampered by a lack of definitively identifying retinal stem cells. Therefore, the present review primarily focuses on current studies that are directed toward identifying endogenous retinal stem cells at the cellular level and recent progress in cellular therapeutic trials using these cells.

\section{THE RETINA AND ENDOGENOUS STEM CELLS}

The retina is composed of nine different neural or glial cells in a highly coordinated manner (Fig. 1). Because of such a sophisticated structure, especially in adult mammals, traditional studies have failed to identify any regenerative potential in the retina after various types of retinal injury. However, further in-depth studies that utilized retinal injury models indicated that cells can differentiate into multiple types of retinal cells in 


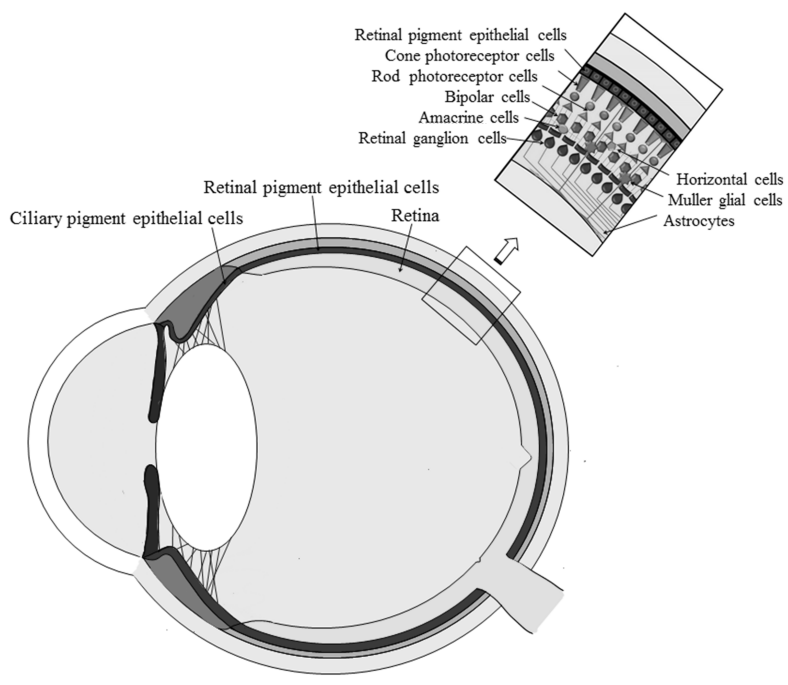

Fig. 1. Anatomy of human eye. Ciliary pigment epithelial cells and retinal pigment epithelial cells are located between retina and choroidal vascular cells. Muller glial cells are located within the retina, extending their cell body vertically throughout the retina.

the injured retina. Therefore, regeneration of the retina is now becoming an emerging possibility.

These endogenous stem cells in the retina include Muller glial cells and ciliary pigment epithelial (CPE) cells, which were identified using injury models of the retina in fish and amphibians. These cells exhibited the potential for retinal regeneration and multi-lineage differentiation, suggesting the possibility that photoreceptor cells derived from these cells may be utilized for transplantation like the clinical transplantation of full-thickness cornea or limbal stem cells. In the present paper, emphasis is placed on the proliferative potential and differentiation potential of several candidate stem cells that are derived from the adult mammalian eye, especially human eye, and the potential of photoreceptor replacement therapy for degenerative retinal diseases.

\section{CELLULAR IDENTITY OF RETINAL STEM CELLS}

\section{Muller glial cells}

Identification of Muller glial cells as a candidate for retinal stem cells: Muller glial cells are the most abundant non-neuronal cells in the retina, providing structural and metabolic support for neural and vascular cells by extending their cell body vertically throughout the retina (10). Muller glial cells share common progenitor cells with other types of retinal neurons (Fig. 2). Muller glial cells have been extensively studied for their potential to regenerate the retina in fish and amphibian eye models (11). However, unlike in fish and amphibians, few studies have identified the significant response of Muller glial cells to retinal insult in mammalian eye models. Instead,

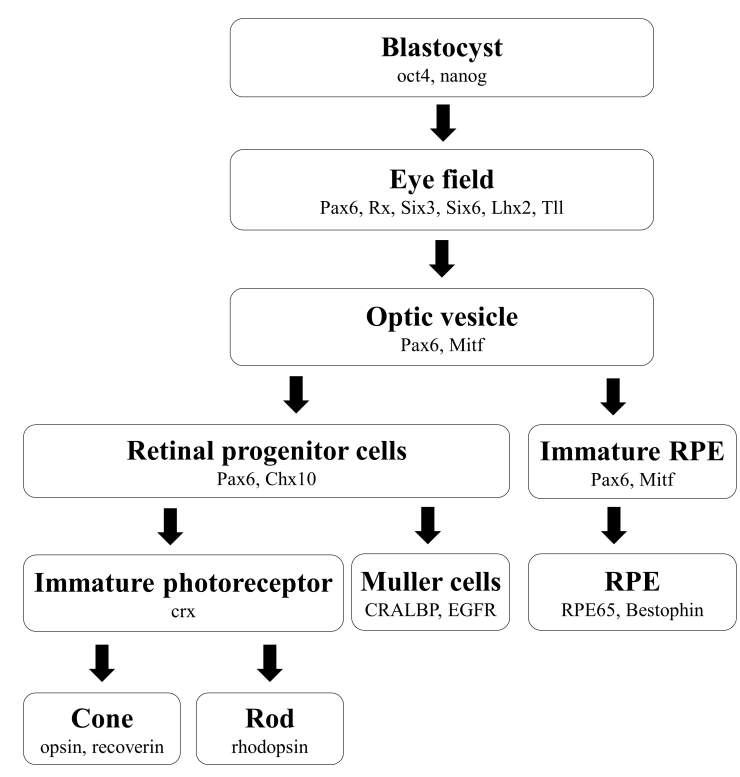

Fig. 2. Stepwise human retinal development and representative markers for retinal commitment.

Muller glial cells were shown to be activated by various kinds of retinal insults and eventually act as a key player in various pathologic processes, especially in retinal proliferative disorders, such as proliferative diabetic retinopathy $(13,14)$, macular holes $(15,16)$, and proliferative vitreoretinopathy $(17,18)$.

However, recent studies have shown that Muller cells in mammals, such as chicks (19) and rats $(20,21)$, may also differentiate into certain retinal cell types, such as cone and rod photoreceptors, raising the possibility that they may play a role as retinal stem cells. Accordingly, studies have focused on identifying and characterizing Muller glial cells in the human retina, but limited access to pure human Muller glial cells and the limited life span of primary Muller glial cells have hindered research (22). Limb and colleagues recently established the immortalized human Muller glial cell line (MIO-M1), the cellular identity of which was verified by its expression of Muller glial cell markers (i.e., glutamine synthetase, $\alpha$-smooth muscle actin, cellular retinaldehyde binding protein [CRALBP], and EGFR [epidermal growth factor receptor]) and electrophysiological response (12). They found that CRALBP- and nestin-positive Muller cells can be immortalized and maintain their characteristics after 50 subculture passages. Subsequent studies revealed that human Muller cells share certain characteristics with neural stem cells, can proliferate more than 20 passages, and express mRNA that encode retinal progenitor markers, such as Sox2, Pax6, Chx10, and Notch1 (5). Moreover, these cells formed a neurosphere when they were plated at a low density (500-800 cells per $\mathrm{cm}^{2}$ ) on an extracellular matrix gel in the presence of fibroblast growth factor-2 (FGF2) or retinoic acid, and the signals played a critical 
role during the early stages of eye development. The clonality of the cells was confirmed by limiting the dilution of the cells and secondary sphere formation. Notably, when these cells were transplanted into subretinal spaces in dystrophic Royal College of Surgeons (RCS) rat and neonatal Lister hooded rats, these cells survived and migrated into a photoreceptor cell layer, inner nuclear layer, and ganglion cell layer. Furthermore, these transplanted cells expressed cell type-specific proteins in each corresponding layer of the retina in neonatal Lister hooded rats but not dystrophic RCS rats, indicating the possible regeneration of retinal tissue under specific physiological conditions (5).

Signals that regulate Muller glial cells: With identification of the potential of retinal regeneration in Muller glial cells, subsequent studies focused on the specific signals that can regulate the differentiation of Muller glial cells. For example, based on the finding that Notch-1 controls the development of retinal ganglion cells in the embryonic retina but needs to cease for the maturation of retinal ganglion cells (23), Becker et al. designed a study to differentiate retinal ganglion cells by blocking Notch-1 (4). The study showed that the inhibition of Notch signaling with $\gamma$-secretase caused human Muller glial cells to acquire neural morphology and express retinal ganglion cell markers, such as Brn3, Isl-1, and Hud (4). Additionally, they showed that Muller glial cells can differentiate into photoreceptor cells when cultured on basement membrane protein in the presence of FGF2, taurine, retinoic acid, and insulin-like growth factor-1, and these cells expressed photoreceptor markers, such as Crx, nuclear receptor subfamily 2, group $\mathrm{E}$, member 3 (NR2E3), rhodopsin, and recoverin (7).

Notably, the same study demonstrated the therapeutic potential of Muller glial cells to recover retinal function in a retinal disease model (i.e., when Muller glial cell-derived photoreceptor cells were transplanted into the retina in 3-week-old $\mathrm{P} 23 \mathrm{H}$ rats, a model of retinitis pigmentosa). The transplanted cells exhibited migration and integration into the outer nuclear layer of the degenerated retinas, leading to improvement in rod photoreceptor function, reflected by an increase in the a-wave amplitude and slope using scotopic flash electroretinography (7).

Collectively, the evidence suggests that human Muller glial cells can be stimulated by injury, proliferate in vitro, differentiate into photoreceptor cells, and, most importantly, be integrated into retinal tissue for the partial restoration of visual function. Although these findings suggest the possibility that Muller glial cells can be used for retinal regeneration, studies on human Muller glial cells are still limited, primarily because of difficulty culturing primary human Muller glial cells.

\section{Ciliary pigment epithelial cells}

Controversy on ciliary pigment epithelial cells as retinal stem cells: The ciliary margins in some non-mammalian vertebrate species have been thought to have stem cells that facilitate neurogenesis throughout life (24). However, much controversy has existed with regard to the possibility that pigment ciliary epithelial cells serve as retinal stem cells in the human eye. Recently, Tropepe et al. found that pigmented CPE cells from mouse and human eyes can form spheres based on limiting-dilution analysis, and a small number of pigmented cells from dissociated primary spheres generated new secondary spheres, giving rise to subsequent colonies in up to six passages $(8,25)$. Moreover, CPE cells within the sphere were positive for Chx10 and nestin (i.e., markers of retinal progenitor cells). Similarly, sphere-forming CPE cells could differentiate into various types of retinal cells that express markers of bipolar cells, rod photoreceptor cells, amacrine cells, and Muller glial cells. Furthermore, studies examined the in vivo regeneration potential of CPE cells by transplanting cells labeled with green fluorescent protein (GFP) into the vitreous cavity in NOD/SCID mice on postnatal day 1 (i.e., a time when photoreceptors and Muller glia are maximally induced to differentiate in the host eye). Among the 16 mice that received the transplant, 12 had GFP-positive cells 28 days after transplantation, and eight mice showed morphological integration and migration into the distal outer layer of the neural retina and RPE where these integrated cells expressed Rom1, a rod photoreceptor outer segment protein (8).

Although these studies raise the exciting possibility that a subpopulation of human CPE cells are retinal stem cells, unresolved issues need to be considered, including the rarity of retinal stem cells (only $0.018 \%$ of single pigmented cells can give rise to clonal colonies), low yield of terminal differentiated cells, and limited potential for integration into the adult mammalian retina. Evidences against the possibility that CPE cells can differentiate into retinal stem cells have also been reported. For example, Cicero et al. argued that human and mouse CPE cells were not stem cells; instead, these cells contain a population of differentiated pigment CPE cells that can proliferate, clonally expand, and self-renew in stem cell medium and express some neuronal markers while retaining features of pigment CPE cells (33). Moreover, adult mammalian CPE cells were reported to have a limited expansion potential, contrary to neural stem cells $(9,27)$. Similarly, Gualdoni et al. expanded rodent CPE cells and evaluated the differentiation potential of photoreceptor cells but failed to differentiate these cells into photoreceptor cells (27). Moreover, Bhatia et al. studied potential retinal stem cells in the human eye by investigating the in situ anatomical distribution of retinal progenitor cell markers. They found that Nestin-positive cells were detected only in the neural retina, which were adjacent to ora serrata, and they expressed Sox2, shh, and chx10 but were not found in ciliary epithelial cells, where only vimentin and Sox2 were expressed (35).

Notably, CPE cells did not express Chx10 when they were plated under adherent conditions (34). This finding is different from the finding in Muller cells, in which Chx10 expression was observed in adherent cultures (5). However, the retinal progenitor markers Chx10 and Rx were expressed only when 
CPE cells were cultured under suspension culture conditions $(34,36)$. Recent studies showed that somatic cells can be at least partially reprogrammed during the spheroid formation process (37). Therefore, the finding that Chx10 is expressed only in sphere cultures of CPE cells requires further confirmation to exclude the possibility that the suspension culture itself has partial reprogramming effects on CPE cells. Additionally, further studies are needed to determine whether the cells in the regenerating retina directly differentiate from retinal stem cells or are trans-differentiated from cultured primary cells.

Limitation of the potential of CPE cells as retinal stem cells: In addition to these issues on the cellular identity of CPE cells, discrepancies have been noted in the clonal proliferation and differentiation potential of various types of retinal cells. For example, one of the major discrepancies concerns the potential of human CPE cells to differentiate into rod photoreceptor cells. Subsequent research by numerous investigators adopted this criterion and reported various ranges of photoreceptor cell differentiation from CPE cells (28-32). Ballios et al. reported that $95 \%$ of the cells differentiated into rhodopsin-positive cells, detected by immunostaining after 44 days of differentiation with retinoic acid and taurine (9). In contrast, only $1 \%$ of opsin-positive cells were generated from rat CPE-derived cells (26), and no fully differentiated photoreceptor cells were generated from porcine CPE-derived cells (27). Gualdoni et al. used Nrl-GFP transgenic mice and failed to effectively activate the $\mathrm{Nrl}$-regulated photoreceptor differentiation program. No rod photoreceptor cells were evident after 2 weeks of differentiation using any previously reported protocol (34). They found that CPE cells were reversed toward the epithelial phenotype when cultured in a retinal differentiation medium and did not differentiate into photoreceptors. Similarly, they found that the transduction of any single transcription factor gene or multiple transcription factors also failed to generate Nrl-GFP-positive cells. These findings were consistent with data reported by Cicero et al., in which no rod cell markers were detected after 3 weeks of differentiation with retinoic acid and taurine (33).

The reason for this discrepancy is not yet clear. However, it could be based on the detection method, and the duration of culture for differentiation into photoreceptors could also be a factor, especially considering that the developmental maturation of rod photoreceptor cells occurs at very late stages of development compared with other retinal cell types. Similarly, the process for ESCs or iPSCs to differentiate into mature rod photoreceptor cells requires a differentiation period of up to 180 days $(38,39)$. However, findings of the expression of immature cell markers of spheroid CPE cells with a failure to produce mature rod photoreceptor cells have been common across studies, indicating a limitation in their potential to differentiate into retinal stem cells. Additionally, the in vivo integration and differentiation observed in NOD/SCID mice also needs to be examined further because the findings were lim- ited to postnatal day $1 \mathrm{NOD} / \mathrm{SCID}$ mice, and such results were not evident in an adult dystrophic RCS model. Similarly, additional studies on transplanted cells that migrate to and are integrated in the retina are warranted to examine their integrated function in the retina. Collectively, human CPE cells can express a certain range of retinal progenitor markers in spheroid form but appear to have limited potential for retinal neuronal differentiation and self-renewal. However, further studies are needed to determine whether these cells can differentiate into retinal neurons using alternative methods or longer periods of differentiation.

\section{Retinal pigment epithelial cells}

Identification of retinal pigment epithelial cells as retinal stem cells: Retinal pigment epithelial cells are pigment epithelial cells that are located between retina and choroidal vascular cells. Retinal pigment epithelial cells play an essential role in homeostasis and phototransduction in the retina (40). These cells are the most widely studied cell types in the retina because of their long history of being used in primary cell cultures. Accordingly, studies that use RPE cells from many species demonstrated their potential for differentiation into retinal ganglion, amacrine, photoreceptor, and glial cells (41-43). For example, Carr et al. evaluated the differentiation potential of RPE cells by culturing them with retinoic acid (44). They found that both ARPE-19 cells (i.e., an immortalized RPE cell line) and passage 2 primary human RPE cells expressed transcripts that were specific for cone photoreceptor cells (red opsin) and the retinal ganglion cell marker Brn3 after 7 days of culture with retinoic acid but failed to express rhodopsin, blue opsin, or interphotoreceptor retinoid binding protein (IRBP).

As observed in other types of retinal progenitor cells, RPE cells that are cultured on a spheroid form tend to exhibit a higher stem cell phenotype. Salero et al. reported that a subgroup of RPE cells was activated into multipotent stem cells with the capacity of self-renewal and differentiation into RPE progeny or neural, osteo, chondro, or adipo-lineage mesenchymal progeny (45). In their report, similar to CPE cells, RPE cells grew more successfully when plated under adherent conditions than under suspension conditions. They found that cultured RPE cells under adherent conditions expressed the early eyefield and forebrain marker Pax6 and RPE marker MITF, but the retinal progenitor markers $\mathrm{CHX} 10$ and rhodopsin were not detected. In contrast, when cultured in suspension, a clonal line of RPE cells can produce RPE, adipocyte, chondrocyte, and bone lineage progeny. Because these studies focused on the transition of RPE cells to mesenchymal progeny, photoreceptor cell fate was not examined. Nonetheless, they showed that RPE spheres expressed early eyefield and forebrain markers, thus demonstrating the possibility of differentiating into retinal neurons. Interestingly, Yan et al. demonstrated that the human RPE cell lines ARPE-19 and hTERT-RPE1 can express photoreceptor proteins, such as IRBP, recoverin, arrestin, transducin, Crx, and red opsin, but not rod 
Table 1. Cellular identity of retinal stem cells

\begin{tabular}{|c|c|c|c|}
\hline & Muller Glial Cells & Ciliary Pigment Epithelial Cells & Retinal Pigment Epithelial Cells \\
\hline \multicolumn{4}{|l|}{ Clonality* } \\
\hline & Yes (5) & Yes $(8,9,33)$ or Minimal $(34)$ & Yes (45) \\
\hline \multicolumn{4}{|c|}{ Expression of retinal progenitor markers } \\
\hline Adherent & Nestin, Pax6, Sox2 and Chx10 (5) & Lhx2, Pax6, Sox2 and Nestin (34) & $\begin{array}{l}\text { Pax6 (44, 45), Crx (44), Nrl } \\
\text { (44), and Mitf (45) }\end{array}$ \\
\hline Sphere & Nestin, Sox2, Pax6, Chx10 and shh (5) & Nestin (33), Chx10 (34) & $\mathrm{n} / \mathrm{a}$ \\
\hline \multicolumn{4}{|c|}{ Differentiation potential } \\
\hline & $\begin{array}{l}\text { Retinal ganglion cells }(3,4) \\
\text { Cone and rod photoreceptor cells }(7)\end{array}$ & $\begin{array}{l}\text { Rod photoreceptor cells }(8,9) \\
\text { Not detected }(33,34)\end{array}$ & Cone photoreceptor cells (44) \\
\hline \multicolumn{4}{|l|}{ Transplantation } \\
\hline Integration & $\begin{array}{l}\text { Retinal ganglion cells in a } 4 \text { week old rat glauco- } \\
\text { ma model ( } 3 \text { ) } \\
\text { Muller glial cells in neonatal Lister hooded rats (5) } \\
\text { Rod photoreceptor precursors in a } 3 \text { week old rat } \\
\text { RP model (7) }\end{array}$ & $\begin{array}{l}\text { Spheres in postnatal day } 1 \mathrm{NOD} / \\
\text { SCID mice (8) } \\
\text { Spheres in newborn albino rat (33) }\end{array}$ & $\mathrm{n} / \mathrm{a}$ \\
\hline Functional recovery & $\begin{array}{l}\text { Improvement of retinal ganglion cells function in } \\
\text { ERG (3) } \\
\text { Improvement of rod photoreceptor function in } \\
\text { ERG (7) }\end{array}$ & $\mathrm{n} / \mathrm{a}$ & $\mathrm{n} / \mathrm{a}$ \\
\hline
\end{tabular}

*Clonality was confirmed by limiting dilution assay and secondary sphere formation assay.

photoreceptor markers when engineered to express neuroD or ngn1 (46).

Although the differentiation potential of RPE cells into photoreceptor cells has not been fully explored, relatively easy acquisition and culture in vitro highlight the possibility of using RPE cells as a source for transplantation in degenerative retinal degeneration.

\section{SUMMARY AND FUTURE DIRECTIONS}

Therapeutic approaches to retinal regeneration are highly warranted. The direct differentiation of pluripotent stem cells, such as ESCs and iPSCs, constitutes one promising approach, but many issues need to be resolved before they can be applied in clinical-grade cell therapy, including biological risk and technical difficulties associated with differentiation culture procedures. The use of endogenous retinal stem cells is another promising approach, in which cell therapy for retinal disease and targeting their microenvironment to trigger their own regenerative potential can be developed. However, more studies are needed to elucidate the precise cellular identity of retinal stem cells that are responsible for retinal regeneration (Table 1). Moreover, the cellular phenomena that underlie retinal regeneration require further in-depth examination because changes in cell fate can be induced, making interpretations of data on retinal differentiation and histological integration in the retina difficult. The differentiation potential of candidate retinal stem cells, such as Muller glial cells, CPE cells, and RPE cells, should be further characterized under different conditions that have not yet been tested. Moreover, their physio- logical significance in the retina should be better defined with regard to their role in retinal regeneration under injury conditions. Studies on the in vivo trafficking of cell fate and behavior under various types of injury stress or homeostatic conditions should further delineate the regeneration process in the retina. Further understanding of the retinal regeneration phenomenon will shed light on the cellular basis of retinal regeneration and expand the horizon for cell therapy for many intractable retinal degenerative diseases.

\section{ACKNOWLEDGEMENTS}

This research was supported by the Korean Government (MEST) and National Agenda Project (NAP, 2009)

\section{REFERENCES}

1. Sohocki MM, Daiger SP, Bowne SJ et al (2001) Prevalence of mutations causing retinitis pigmentosa and other inherited retinopathies. Hum Mutat 17, 42-51

2. Schwartz SD, Hubschman JP, Heilwell G et al (2012) Embryonic stem cell trials for macular degeneration: a preliminary report. Lancet 379, 713-720

3. Singhal S, Bhatia B, Jayaram H et al (2012) Human Müller glia with stem cell characteristics differentiate into retinal ganglion cell (RGC) precursors in vitro and partially restore RGC function in vivo following transplantation. Stem Cells Transl Med 1, 188-199

4. Becker S, Singhal S, Jones MF et al (2013) Acquisition of RGC phenotype in human Müller glia with stem cell characteristics is accompanied by upregulation of functional 
nicotinic acetylcholine receptors. Mol Vis 19, 1925-1936

5. Lawrence JM, Singhal S, Bhatia B et al (2007) MIO-M1 cells and similar muller glial cell lines derived from adult human retina exhibit neural stem cell characteristics. Stem Cells 25, 2033-2043

6. Giannelli SG, Demontis GC, Pertile G, Rama P and Broccoli V (2011) Human Müller glia cells are a highly efficient source of rod photoreceptors. Stem Cells 29, 344-356

7. Jayaram H, Jones MF, Eastlake K et al (2014) Transplantation of photoreceptors derived from human Muller glia restore rod function in the $\mathrm{P} 23 \mathrm{H}$ rat. Stem Cells Transl Med 3, 323-333

8. Coles BL, Angénieux B, Inoue T et al (2004) Facile isolation and the characterization of human retinal stem cells. Proc Natl Acad Sci U S A 101, 15772-15777

9. Ballios BG, Clarke L, Coles BL, Shoichet MS and Van Der Kooy D (2012) The adult retinal stem cell is a rare cell in the ciliary epithelium whose progeny can differentiate into photoreceptors. Biol Open 1, 237-246

10. Turner DL and Cepko CL (1987) A common progenitor for neurons and glia persists in rat retina late in development. Nature 328, 131-136

11. Bernardos RL, Barthel LK, Meyers JR and Raymond PA (2007) Late-stage neuronal progenitors in the retina are radial Müller glia that function as retinal stem cells. J Neurosci 27, 7028-7040

12. Limb GA, Salt TE, Munro PM, Moss SE and Khaw PT (2002) In vitro characterization of a spontaneously immortalized human Müller cell line (MIO-M1). Invest Ophthalmol Vis Sci 43, 864-869

13. Nork TM, Wallow IH, Sramek SJ and Anderson G (1987) Muller's cell involvement in proliferative diabetic retinopathy. Arch Ophthalmol 105, 1424-1429

14. Mizutani M, Gerhardinger C and Lorenzi M (1998) Muller cell changes in human diabetic retinopathy. Diabetes 47 , 445-449

15. Ezra E, Munro PM, Charteris DG, Aylward WG, Luthert PJ and Gregor ZJ (1997) Macular hole opercula: ultrastructural features and clinicopathological correlation. Arch Ophthalmol 115, 1381-1387

16. Messmer EM, Heidenkummer HP and Kampik A (1998) Ultrastructure of epiretinal membranes associated with macular holes. Graefes Arch Clin Exp Ophthalmol 236, 248-254

17. Lewis GP, Erickson PA, Guérin CJ, Anderson DH and Fisher SK (1989) Changes in the expression of specific Müller cell proteins during long-term retinal detachment. Exp Eye Res 49, 93-111

18. McGillem GS and Dacheux RF (1999) Rabbit retinal Muller cells undergo antigenic changes in response to experimentally induced proliferative vitreoretinopathy. Exp Eye Res 68, 617-627

19. Fischer AJ and Reh TA (2001) Müller glia are a potential source of neural regeneration in the postnatal chicken retina. Nat Neurosci 4, 247-252

20. Ooto S, Akagi T, Kageyama R et al (2004) Potential for neural regeneration after neurotoxic injury in the adult mammalian retina. Proc Natl Acad Sci U S A 101, 13654-13659

21. Das AV, Mallya KB, Zhao X et al (2006) Neural stem cell properties of Müller glia in the mammalian retina: regulation by Notch and Wnt signaling. Dev Biol 299, 283-302

22. Sarthy VP, Brodjian SJ, Dutt K, Kennedy BN, French RP and Crabb JW (1998) Establishment and characterization of a retinal Muller cell line. Invest Ophthalmol Vis Sci 39, 212-216

23. Austin CP, Feldman DE, Ida JA Jr and Cepko CL (1995) Vertebrate retinal ganglion cells are selected from competent progenitors by the action of Notch. Development $121,3637-3650$

24. Coulombre JL and Coulombre AJ (1965) Regeneration of neural retina from the pigmented epithelium in the chick embryo. Dev Biol 12, 79-92

25. Tropepe V, Coles BL, Chiasson BJ et al (2000) Retinal stem cells in the adult mammalian eye. Science 287, 2032-2036

26. Das AV, James J, Rahnenführer J et al (2005) Retinal properties and potential of the adult mammalian ciliary epithelium stem cells. Vision Res 45, 1653-1666

27. MacNeil A, Pearson RA, MacLaren RE, Smith AJ, Sowden JC and Ali RR (2007) Comparative analysis of progenitor cells isolated from the iris, pars plana, and ciliary body of the adult porcine eye. Stem Cells 25, 2430-2438

28. Akagi T, Mandai M, Ooto S et al (2004) Otx2 homeobox gene induces photoreceptor-specific phenotypes in cells derived from adult iris and ciliary tissue. Invest Ophthalmol Vis Sci 45, 4570-4575

29. Jomary C, Jones SE and Lotery AJ (2010) Generation of light-sensitive photoreceptor phenotypes by genetic modification of human adult ocular stem cells using Crx. Invest Ophthalmol Vis Sci 51, 1181-1189

30. Jomary C and Jones SE (2008) Induction of functional photoreceptor phenotype by exogenous $\mathrm{Crx}$ expression in mouse retinal stem cells. Invest Ophthalmol Vis Sci 49, 429-437

31. Giordano F, De Marzo A, Vetrini F and Marigo V (2007) Fibroblast growth factor and epidermal growth factor differently affect differentiation of murine retinal stem cells in vitro. Mol Vis 13, 1842-1850

32. Djojosubroto M, Bollotte F, Wirapati P, Radtke F, Stamenkovic I and Arsenijevic Y (2009) Chromosomal number aberrations and transformation in adult mouse retinal stem cells in vitro. Invest Ophthalmol Vis Sci 50, 5975-5987

33. Cicero SA, Johnson D, Reyntjens S et al (2009) Cells previously identified as retinal stem cells are pigmented ciliary epithelial cells. Proc Natl Acad Sci U S A 106, 6685-6690

34. Gualdoni S, Baron M, Lakowski J et al (2010) Adult ciliary epithelial cells, previously identified as retinal stem cells with potential for retinal repair, fail to differentiate into new rod photoreceptors. Stem Cells 28, 1048-1059

35. Bhatia B, Singhal S, Lawrence JM, Khaw PT and Limb GA (2009) Distribution of Müller stem cells within the neural retina: evidence for the existence of a ciliary margin-like zone in the adult human eye. Exp Eye Res 89, 373-382

36. Kokkinopoulos I, Pearson RA, Macneil A et al (2008) Isolation and characterization of neural progenitor cells from the adult Chx10 (orj/orj) central neural retina. Mol Cell Neurosci 38, 359-373 
37. Liu Y, Mukhopadhyay P, Pisano MM et al (2013) Repression of Zeb1 and hypoxia cause sequential mesenchymal-to-epithelial transition and induction of aid, Oct4, and Dnmt1, leading to immortalization and multipotential reprogramming of fibroblasts in spheres. Stem Cells 31, 1350-1362

38. Hirami Y, Osakada F, Takahashi K et al (2009) Generation of retinal cells from mouse and human induced pluripotent stem cells. Neurosci Lett 458, 126-131

39. Meyer JS, Shearer RL, Capowski EE et al (2009) Modeling early retinal development with human embryonic and induced pluripotent stem cells. Proc Natl Acad Sci U S A $106,16698-16703$

40. Strauss $O$ (2005) The retinal pigment epithelium in visual function. Physiol Rev 85, 845-881

41. Zhao S, Thornquist SC and Barnstable CJ (1995) In vitro transdifferentiation of embryonic rat retinal pigment epithelium to neural retina. Brain Res 677, 300-310

42. Ma W, Yan RT, Xie W and Wang SZ (2004) bHLH genes cath5 and cNSCL1 promote bFGF-stimulated RPE cells to transdifferentiate toward retinal ganglion cells. Dev Biol 265, 320-328

43. Sakaguchi DS, Janick LM and Reh TA (1997) Basic fibroblast growth factor (FGF-2) induced transdifferentiation of retinal pigment epithelium: generation of retinal neurons and glia. Dev Dyn 209, 387-398

44. Carr AJ, Vugler AA, Yu L et al (2011) The expression of retinal cell markers in human retinal pigment epithelial cells and their augmentation by the synthetic retinoid fenretinide. Mol Vis 17, 1701-1715

45. Salero E, Blenkinsop TA, Corneo B et al (2012) Adult human RPE can be activated into a multipotent stem cell that produces mesenchymal derivatives. Cell Stem Cell 10, 88-95

46. Yan RT, Li X, Huang J, Guidry C and Wang SZ (2013) Photoreceptor-like cells from reprogramming cultured mammalian RPE cells. Mol Vis 19, 1178-1187 